\title{
Expression of dentin matrix protein 1 in tumors causing oncogenic osteomalacia
}

\author{
Satoru Toyosawa ${ }^{1}$, Yasuhiko Tomita ${ }^{2}$, Mitsunobu Kishino ${ }^{1}$, Jun Hashimoto ${ }^{3}$, Takafumi \\ Ueda $^{3}$, Takahiro Tsujimura ${ }^{4}$, Katsuyuki Aozasa ${ }^{2}$, Naokuni Ijuhin ${ }^{1}$ and Toshihisa Komori ${ }^{5}$, \\ Osaka Pathology Conference \\ ${ }^{1}$ Department of Oral Pathology, Osaka University Graduate School of Dentistry, Suita, Osaka, Japan; \\ ${ }^{2}$ Department of Pathology; ${ }^{3}$ Department of Orthopedic Surgery, Osaka University Graduate School of \\ Medicine, Suita, Osaka, Japan; ${ }^{4}$ Division of Pathology, Sumitomo Hospital, Osaka, Osaka, Japan and \\ ${ }^{5}$ Department of Molecular Medicine, Osaka University Graduate School of Medicine, Suita, Osaka, Japan
}

\begin{abstract}
Oncogenic osteomalacia, which is characterized by renal phosphate wasting, low serum 1, 25-dihydroxyvitamin $D$, and osteomalacia, is caused by mesenchymal neoplasms that are termed phosphaturic mesenchymal tumors (PMTs). As PMTs are usually small and lack specific histological features, the pathological identification of PMTs is difficult. Dentin matrix protein 1 (DMP1) is an acidic phosphoprotein expressed in mineralized tissues including bone, tooth, and hypertrophic cartilage. Increased expression of DMP1 gene in PMTs has been reported by using differential cDNA screening. In the present study, DMP1 expression in PMTs and other soft tissue tumors was analyzed immunohistochemically to verify its utility in the differential diagnosis of PMTs. Anti-DMP1 polyclonal antibody was raised against the C-terminal sequence of DMP1. Three cases with PMTs and 11 other soft tissue tumors (two malignant hemangiopericytomas, three solitary fibrous tumors, three synovial sarcomas, and three malignant peripheral nerve sheath tumors) were analyzed for DMP1 expression. DMP1 expression was observed in all of the three cases with PMTs, but never found in other soft tissue tumors examined. DMP1 was detected in the extracellular matrix with myxomatous features or around capillary vessels, and in dystrophic calcified sites. Paranuclear DMP1 staining in the tumor cells was also observed. These findings indicate that DMP1 immunohistochemistry is a useful tool for identifying PMTs.
\end{abstract}

Modern Pathology (2004) 17, 573-578, advance online publication, 5 March 2004; doi:10.1038/modpathol.3800084

Keywords: dentin matrix protein 1; oncogenic osteomalacia; soft tissue tumor; differential diagnosis; immunohistochemistry

Oncogenic osteomalacia is a hypophosphatemic disease, which is characterized by renal phosphate wasting, low serum 1, 25-dihydroxyvitamin D, and osteomalacia, and is caused by neoplasms. ${ }^{1-3}$ The causative neoplasms are termed phosphaturic mesenchymal tumors (PMTs), because these mesenchymal tumors produce renal phosphaturic factors. Clinically, the oncogenic osteomalacia patients suffer from bone pain, bone fractures, and general fatigue, and may finally become bedridden with severe proximal myopathy, mimicking neuromuscular diseases. The tumorous lesions may not be noticed because they are usually small at first presentation and occur in peculiar locations such

Correspondence: Dr S Toyosawa, DDS, PhD, Department of Oral Pathology, Osaka University Graduate School of Dentistry, 1-8 Yamadaoka, Suita, Osaka 565-0871, Japan.

E-mail: toyosawa@dent.osaka-u.ac.jp

Received 21 March 2003; revised 01 October 2003; accepted 06 October 2003; published online 5 March 2004 as a big toe. ${ }^{4}$ Biochemical and skeletal defects in oncogenic osteomalacia are caused by humoral factors produced by the tumors. In fact, surgical removal of the tumor tissue results in enhanced renal conservation of phosphate accompanied by normalization of serum phosphate and 1, 25-dihydroxyvitamin $\mathrm{D}$ levels and subsequent remineralization of bone. Microscopically, PMTs show a wide variety of histologic features without specific characteristics, ${ }^{1,2}$ although most PMTs present as benign mesenchymal tumors with complex vascular network, i.e., hemangiopericytoma-like tumors. ${ }^{4}$

Dentin matrix protein 1 (DMP1) is an acidic phosphoprotein originally identified from a rat incisor cDNA library. ${ }^{5}$ DMP1 is expressed in mineralized tissues including bone, tooth, and hypertrophic cartilage. ${ }^{6-10}$ Recombinant DMP1 promotes hydroxyapatite formation in a gelatin gel system, ${ }^{11}$ and overexpression of DMP1 accelerates the onset of mineralization in both MC3T3-E1 and C3H10T1/2 cells and increases the size of the mineralized 
nodules. ${ }^{12}$ Therefore, DMP1 is supposed to bind to calcium and regulate matrix mineralization.

Recently, increased expression of DMP1 gene in PMTs was reported by differential cDNA screening. ${ }^{13,14}$ We postulated that elevated expression of DMP1 might be observed among the cases of PMTs but not in other soft tissue tumors, and evaluated DMP1 expression immunohistochemically in three cases of PMTs together with 11 other soft tissue tumors in order to clarify the diagnostic utility of DMP1 expression in these lesions.

\section{Materials and methods}

\section{Patients}

Three patients with oncogenic osteomalacia were included in the present study. One patient was a 54year-old woman who was admitted to Osaka University Hospital complaining of bone pain and muscle weakness. She showed typical features of hypophosphatemic osteomalacia and had a small intradermal tumor in her left hip. The clinical courses of the other two patients have been described elsewhere. ${ }^{15,16}$ Briefly, they also showed typical features of hypophosphatemic osteomalacia. One patient had a small soft tissue tumor in the lower mediastinum and the other patient had a small nasal tumor. After surgical removal of the tumors, all the three patients recovered completely from their symptoms including the low serum phosphate. Tissue samples used in this study were obtained in compliance with the guidelines of the Joint Commission on Clinical Investigation of Osaka University Graduate School of Medicine. In addition to the tumor specimens obtained from the three patients with oncogenic osteomalacia, two malignant hemangiopericytomas, three solitary fibrous tumors, three synovial sarcomas, and three malignant peripheral nerve sheath tumors were enrolled for the present analysis. Surgically resected specimens were fixed in $10 \%$ formalin and routinely processed for paraffin embedding. Histologic sections cut at $5 \mu \mathrm{m}$ were stained with hematoxylin and eosin and immunoperoxidase procedures (avidinbiotin Complex (ABC) method). Brief clinical features in these cases such as age, gender, and tumor location are summarized in Table 1.

\section{DMP1 Antibody Production}

Peptide C sequence encoding DMP1 residues (DAYHNKPIGDQDDND) shared the C-termini of human, bovine, rat, and mouse DMP1s (Figure 1). The peptide $\mathrm{C}$ was prepared with $\mathrm{NH}_{2}$-terminal cysteines on the ABI 433A peptide synthesizer (Applied Biosystems, Foster City, CA, USA). Purity of the synthesized peptide was confirmed by highperformance liquid chromatography and amino acid analysis. The cysteinyl peptides were coupled to keyhole limpet hemocyanin and dialyzed, and mixtures of coupled and uncoupled peptides were mixed with TiterMax Gold (CytRx Co., Norcross, GA, USA) and injected subcutaneously into New Zealand white rabbits. Boosting injections were administered with TiterMax Gold. Anti-DMP1 (peptide $\mathrm{C}$ ) antisera were affinity purified by acetic acid elution from a peptide-conjugated SulfoLink Column (Pierce, Rockford, IL, USA).

\section{Immunoblotting}

To characterize the anti-DMP1 (peptide C) antibody, recombinant rat $\mathrm{DMP}^{9}{ }^{9}$ was used for the immunoblots. The recombinant rat DMP1 was separated by

Table 1 Clinical summaries of PMT cases and other soft tissue tumors examined

\begin{tabular}{|c|c|c|c|c|c|}
\hline Case & Age/sex (years) & Location & Diagnosis & $\begin{array}{c}\text { Osteomalacia } \\
\text { hypophosphatemia }\end{array}$ & $\begin{array}{l}\text { Recovery of symptom } \\
\text { after tumor resection }\end{array}$ \\
\hline 1 & $53 / \mathrm{F}$ & Left hip & PMT & + & Yes \\
\hline 2 & $54 / \mathrm{M}$ & Lower mediastinum & PMT & + & Yes \\
\hline 3 & $53 / F$ & Nasal cavity & PMT & + & Yes \\
\hline 4 & $88 / F$ & Upper leg & Malignant hemangiopericytoma & - & / \\
\hline 5 & $67 / \mathrm{M}$ & Forearm & Malignant hemangiopericytoma & - & l \\
\hline 6 & $34 / F$ & Abdominal wall & Solitary fibrous tumor & - & I \\
\hline 7 & $61 / \mathrm{F}$ & Pleura & Solitary fibrous tumor & - & / \\
\hline 8 & $31 / \mathrm{F}$ & Nasal cavity & Solitary fibrous tumor & - & l \\
\hline 9 & $36 / \mathrm{M}$ & Buttock & Synovial sarcoma & - & / \\
\hline 10 & $25 / \mathrm{F}$ & Abdomen & Synovial sarcoma & - & l \\
\hline 11 & $45 / \mathrm{M}$ & Hand & Synovial sarcoma & - & / \\
\hline 12 & $67 / \mathrm{M}$ & Knee & Malignant peripheral nerve sheath tumor & - & / \\
\hline 13 & $34 / \mathrm{F}$ & Retroperitoneum & Malignant peripheral nerve sheath tumor & - & l \\
\hline 14 & $39 / \mathrm{M}$ & Shoulder & Malignant peripheral nerve sheath tumor & - & l \\
\hline
\end{tabular}




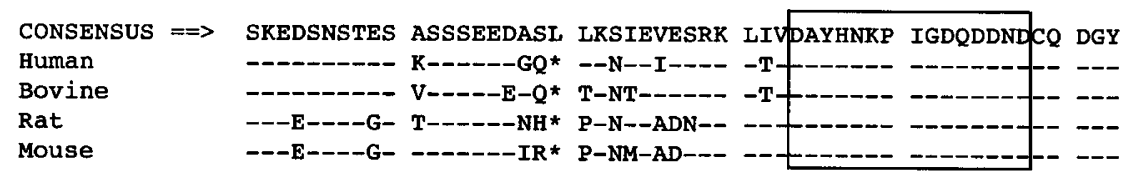

Figure 1 Amino-acid sequence alignment of human, bovine, rat, and mouse DMP1s. Boxed sequences (peptide C), which shared the Cterminals of human, bovine, rat, and mouse DMP1s, are used for the production of the anti-DMP1 (peptide C) antibody. An asterisk ( ${ }^{*}$ ) indicates an alignment gap. Amino-acid residues are given in the IUPAC-IUB single-letter code.

Table 2 Summary of the primary antibodies used in this study

\begin{tabular}{|c|c|c|c|c|}
\hline Antibodies & Source & Tyре & Antigen retrieval & Dilution \\
\hline AE1/AE2 & DakoCytomation, Glostrup, Denmark & Mono (mouse) & Microwave* & $1: 50$ \\
\hline Vimentin (3B4) & DakoCytomation, Glostrup, Denmark & Mono (mouse) & Microwave* & $1: 200$ \\
\hline S-100 & DakoCytomation, Glostrup, Denmark & Poly (rabbit) & Trypsin digestion & $1: 500$ \\
\hline$\alpha$-smooth muscle actin (1A4) & DakoCytomation, Glostrup, Denmark & Mono (mouse) & Microwave* & $1: 50$ \\
\hline CD34 (QBEnd 10) & DakoCytomation, Glostrup, Denmark & Mono (mouse) & Microwave* & $1: 50$ \\
\hline Factor VIII & DakoCytomation, Glostrup, Denmark & Poly (rabbit) & Trypsin digestion & 1:500 \\
\hline Factor XIIIa & Behringwerke, Germany & Poly (rabbit) & Microwave* & $1: 3000$ \\
\hline DMP1 (peptide C) & & Poly (rabbit) & Trypsin digestion & $1: 2000$ \\
\hline
\end{tabular}

${ }^{*}$ Microwaving in $0.1 \mathrm{M}$ citrate buffer.

electrophoresis on sodium dodecyl sulfate-polyacrylamide gel and transferred to polyvinylidene difluoride transfer membranes (Millipore, Bedford, MA, USA) using a semidry transfer system (Nihon Eido, Tokyo, Japan). Membranes were blocked for $1 \mathrm{~h}$ with $5 \%$ bovine serum albumin in Tris-buffered saline with $0.05 \%$ Tween-20 and incubated overnight with anti-DMP1 (peptide C) antibody at a dilution of 1:10000. After applying biotinylated $F$ $\left(\mathrm{ab}^{\prime}\right)_{2}$ fragments of swine anti-rabbit IgG antibody at a dilution of 1:1000 (DakoCytomation, Glostrup, Denmark), the sections were incubated with alkaline phosphatase-conjugated streptavidin at a dilution of 1:500 (DakoCytomation) and then with nitroblue tetrazolium chloride and 5-bromo-4-chloro-3-indolyl-phosphate solution (Roche Diagnostics, Rotkreuz, Switzerland) for $30 \mathrm{~min}$.

\section{Tissue Samples and Immunohistochemistry}

Human and rat normal bone samples were used as positive controls. Bone samples were demineralized in buffered $10 \%$ EDTA for 7 days at $4^{\circ} \mathrm{C}$ with agitation after formalin fixation. After the processing, the bone samples were rinsed and paraffinembedded. Immunoperoxidase procedures (ABC method) with anti-DMP1 (peptide C) and other antibodies were performed on the paraffin-embedded sections. Procedures of antigen retrieval, dilution, and supplier for each primary antibody are listed in Table 2. Sections were lightly counterstained with methylgreen. For negative controls, non-immunized rabbit or mouse IgG serum (DakoCytomation) was used as a primary antibody and uniformly gave negative results.

\section{Results}

\section{Characterization of Anti-DMP1 (peptide C) Antibody}

Anti-DMP1 (peptide C) sera showed specific reaction to DMP1 peptide C by dot blot assay (data not shown). However, affinity purification of the antibody on peptide $\mathrm{C}$ columns was performed prior to its use as an immunoblot reagent. The antibody reacted to $130 \mathrm{kDa}$ recombinant rat DMP1-glutathion-S-transferase (GST) fusion protein and $100 \mathrm{kDa}$ recombinant rat DMP1 protein, in which GST protein had been removed by the digestion with PreScisson Protease (Amersham Biosciences, Piscataway, NJ, USA), whereas preimmune sera showed no reaction (Figure 2(a)). Pericellular bone matrix of osteocytes including their processes in both rat and human bone samples were positively stained with anti-DMP1 (peptide C) antibody (Figures 2(b) and (c)), which is compatible with our previous observation using the antibody against the N-terminus of rat DMP $1 .{ }^{9}$ When the antigen peptide $\mathrm{C}$ was preincubated with the primary antibody, the immunoreactivities disappeared, confirming the specificity of the antibody (data not shown). Thus, the anti-DMP1 (peptide C) antibody was demonstrated to recognize the precise human DMP1 molecules as well as those of rat.

\section{Histological Findings}

Histologically, all three PMTs contained primitiveappearing stromal cells, variably prominent vessels (Figures $3\left(a_{1}\right)$ and $\left(b_{1}\right)$ ) similar to those found in hemangiopericytomas, and/or osteoclast-like giant cells, and thus were classified as mixed connective 

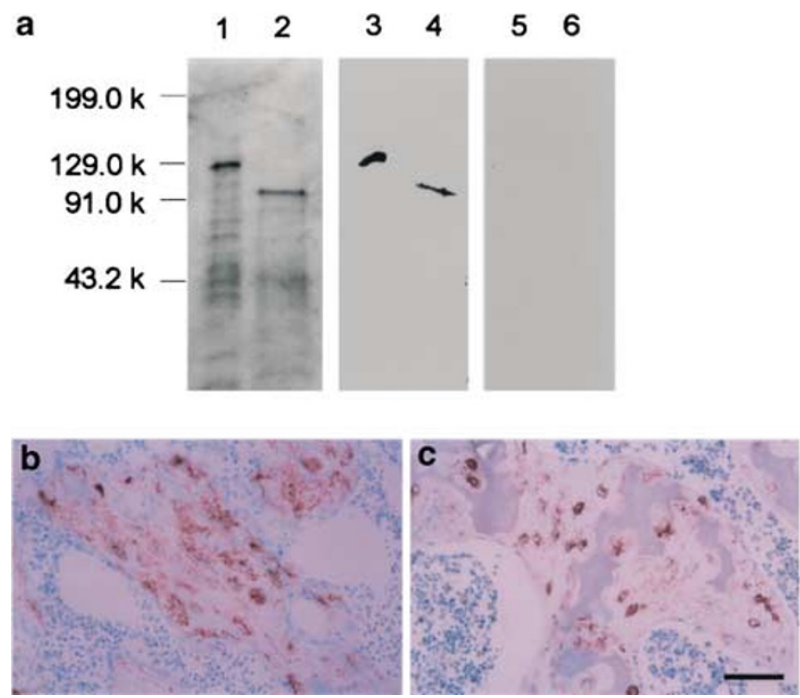

Figure 2 Characterization of the anti-DMP1 (peptide C) antibody (a) Lanes 1, 2: sodium dodecyl sulfate-polyacrylamide gel (7\%) electrophoresis of recombinant rat DMP1 produced in E.coli. Lanes 3-6: immunoblot analysis of the recombinant rat DMP1. Lane 1 shows the recombinant DMP1-glutathione S-transferase (GST) fusion protein. Lane 2 indicates recombinant DMP1 after digestion with PreScisson Protease. The recombinant DMP1-GST fusion protein (lane 3) and the recombinant DMP1 protein (lane 4) are detected using affinity-purified anti-DMP1 (peptide C) antibody, while both recombinant proteins are not detected by preimmune sera (lanes 5, 6). (b, c) Immunohistochemical detection of DMP1 in rat and human bones. Anti-DMP1 (peptide C) antibody stained the pericellular bone matrix of osteocytes including their processes in the metaphysial trabecular bone of both a postnatal 15-day-old rat (b) and a 23-week-old human embryo (c). Methylgreen counterstain, bar $=125 \mu \mathrm{m}$.

tissue tumors. Cartilaginous or myxomatous areas (Figure $3\left(\mathrm{c}_{1}\right)$ ), dystrophic calcification (Figure $3\left(\mathrm{~d}_{1}\right)$ ), focal microcystic changes (Figure $3\left(\mathrm{e}_{1}\right)$ ), and osseous metaplasia were found in all three tumors. The tumor cells lacked cellular pleomorphism, and mitotic figures were rarely seen. These three samples contained some capsular connective tissues that enclosed the tumor cells, which did not invade the surrounding tissues.

\section{Immunohistochemical Profiles of PMTs and Other Soft Tissue Tumors}

The immunohistochemical profiles of PMTs and other soft tissue tumors are listed in Table 3. All PMTs showed positive immunoreactivities for vimentin, CD34, and factor XIIIa expression, similar to malignant hemangiopericytomas (Table 3). Instead, a remarkable difference was observed between the two groups as for DMP1 expression. DMP1 expression was observed in all the three PMTs, but never in the two malignant hemangiopericytomas and other soft tissue tumors examined (Table 3). As DMP1 was demonstrated to be a specific histological marker identifying PMTs, localization of DMP1 in the tumors was further investigated. DMP1 was

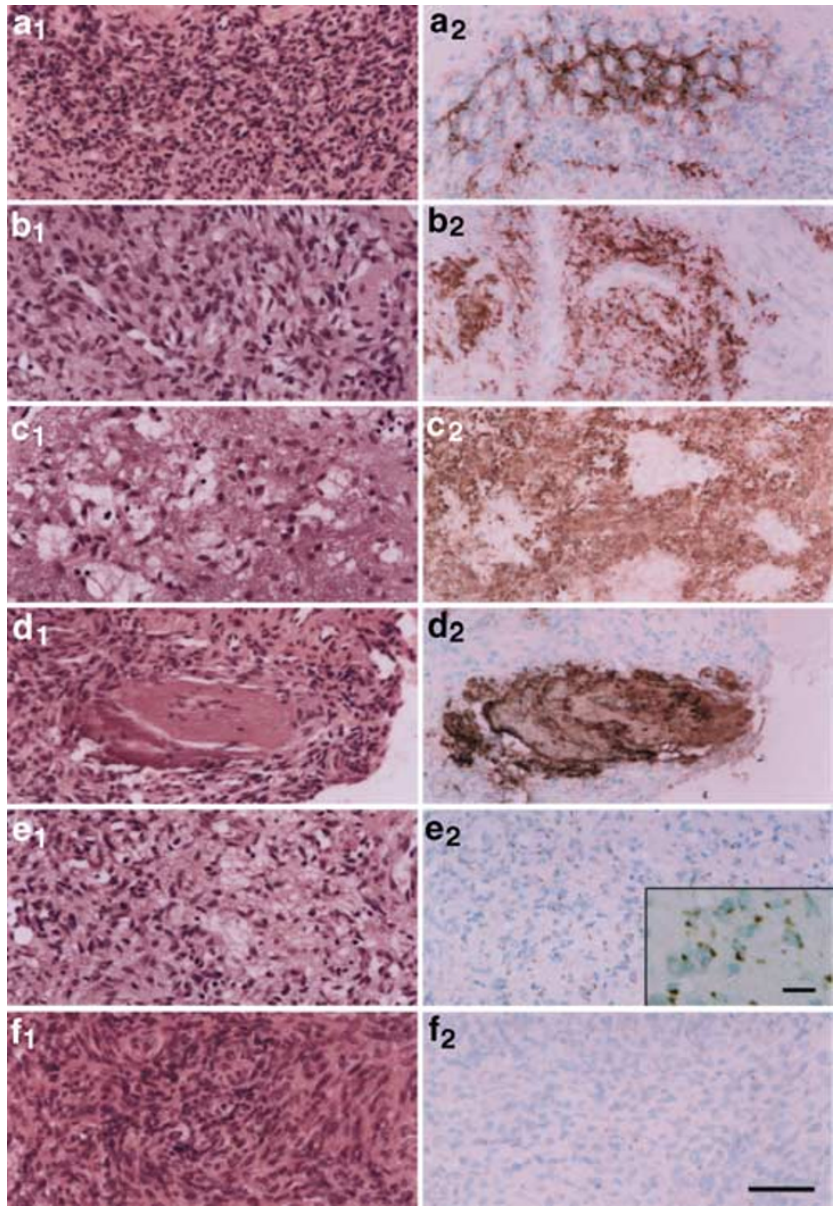

Figure 3 Immunohistochemical localization of DMP1 in the PMTs. The horizontal rows $\mathbf{a}$ and $\mathbf{b}$ show the photographs of cases 1 and 2 with PMTs, respectively. The horizontal rows $\mathbf{c}-\mathbf{f}$ show the photographs of case 3 with PMT. The right vertical column shows DMP1 immunostaining $\left(\mathbf{a}_{2}-\mathbf{f}_{2}\right)$, compared to the left vertical column of H\&E-stained serial sections $\left(a_{\mathbf{1}}-\mathbf{f}_{\mathbf{1}}\right) .\left(\mathbf{a}_{\mathbf{1}}-\mathbf{f}_{\mathbf{1}}\right)$ $\mathrm{H} \& \mathrm{E}$ stain, $\left(\mathbf{a}_{2}-\mathbf{f}_{2}\right)$ methylgreen counterstain, bar $=100 \mu \mathrm{m}$, bar $=15 \mu \mathrm{m}$ in inset of $\left(\mathbf{e}_{2}\right)$. PMTs show a heterogeneous DMP1 staining pattern. (a) Case 1: In the area containing numerous vessels in a hemangiopericytoma-like pattern, strong DMP1 immunostaining is present in the matrix around capillary vessels. (b) Case 2: Granular deposits of DMP1 are present in the matrix around capillary vessels. (c) Case 3: Granular deposits of DMP1 are observed in the cartilage-like or myxomatous stroma. (d) Case 3: DMP1 is also detected in the foci of dystrophic calcification. (e) Case 3: In the area with microcystic changes within the stroma, paranuclear immunostaining of DMP1 is present within the tumor cells. High magnification of paranuclear immunostaining of DMP1 in tumor cells is shown in the inset of $\left(\mathbf{e}_{2}\right)$. (f) Case 3: In the area composed of packed spindle-shaped stromal cells, little immunostaining of DMP1 is detected.

localized in the extracellular matrix around tumor cells (Figures 3(a-d)). Granular deposits of DMP1 were present in the myxomatous stroma (Figure 3(c)) and in the matrix around capillary vessels in the hemangiopericytoma-like areas (Figures 3(a) and (b)). DMP1 was also detected in the foci of dystrophic calcification (Figure 3(d)). Paranuclear staining was observed in the tumor cells in widespread areas including the area with microcystic 
Table 3 Immunohistochemical profiles of PMTs and other soft tissue tumors

\begin{tabular}{|c|c|c|c|c|c|c|c|c|c|c|c|c|c|c|}
\hline \multirow[t]{2}{*}{ Antibodies } & \multicolumn{3}{|c|}{$P M T$} & \multicolumn{2}{|c|}{$\begin{array}{c}\text { Malignant } \\
\text { hemangiopericytoma }\end{array}$} & \multicolumn{3}{|c|}{$\begin{array}{l}\text { Solitary } \\
\text { fibrous } \\
\text { tumor }\end{array}$} & \multicolumn{3}{|c|}{$\begin{array}{l}\text { Synovial } \\
\text { sarcoma }\end{array}$} & \multicolumn{3}{|c|}{$\begin{array}{c}\text { Malignant } \\
\text { peripheral nerve } \\
\text { sheath tumor }\end{array}$} \\
\hline & 1 & 2 & 3 & 4 & 5 & 6 & 7 & 8 & 9 & 10 & 11 & 12 & 13 & 14 \\
\hline AE1/AE3 & - & - & - & - & - & - & - & - & $+\mathrm{F}$ & $+\mathrm{F}$ & $+\mathrm{F}$ & - & - & - \\
\hline Vimentin (3B4) & + & + & + & + & + & + & + & + & + & + & + & + & + & + \\
\hline S-100 & - & - & - & - & - & - & - & - & - & - & - & + & + & + \\
\hline $\begin{array}{l}\alpha \text {-smooth muscle actin } \\
(1 \mathrm{~A} 4)\end{array}$ & $-^{*}$ & $-*$ & $-*$ & $-{ }^{*}$ & $-^{*}$ & $-^{*}$ & $-{ }^{*}$ & $-*$ & $-{ }^{*}$ & $-^{*}$ & $-*$ & $-*$ & $-*$ & $-*$ \\
\hline Factor VIII & $-{ }^{*}$ & $-{ }^{*}$ & $-*$ & $-*$ & $-*$ & $-{ }^{*}$ & $-{ }^{*}$ & $-{ }^{*}$ & - * & $-{ }^{*}$ & $-*$ & $-*$ & $-{ }^{*}$ & -* $^{*}$ \\
\hline Factor XIIIa & $+\mathrm{F}$ & $+\mathrm{F}$ & + & + & + & + & + & + & - & - & - & - & - & - \\
\hline CD34 (QBEnd 10) & $+\mathrm{F}^{*}$ & $+^{*}$ & $+\mathrm{F}^{*}$ & $+^{*}$ & $+\mathrm{F}^{*}$ & $+^{*}$ & $+^{*}$ & $+^{*}$ & $-{ }^{*}$ & $-{ }^{*}$ & $-{ }^{*}$ & $-{ }^{*}$ & $-{ }^{*}$ & - * \\
\hline DMP1 & + & + & + & - & - & - & - & - & - & - & - & - & - & - \\
\hline
\end{tabular}

F:focally; *endothelial cells of the vessels are positive.

changes (Figure 3(e)). Two out of three PMTs contained the focal area composed of packed spindle-shaped cells, in which DMP1 was barely detectable (Figure 3(f)).

\section{Discussion}

From the analysis of gene expression profiles, the modulation of several genes in PMT has been reported, such as matrix extracellular phosphoglycoprotein, fibroblast growth factor-23 (FGF-23), heat shock protein 90, and osteopontin. Among them, a marked increase of $D M P 1$ gene expression was constantly observed in PMTs. ${ }^{13,14}$ To analyze the expression of DMP1 in the PMTs at the protein level and thereafter clarify its diagnostic utility, the present study was employed.

DMP1 expression is restricted to bone, tooth, and hypertrophic cartilage in non-neoplastic tissues, and is supposed to relate to the mineralization process. ${ }^{6-10}$ In bone tissue, DMP1 gene is predominantly expressed in osteocytes, but not in osteoblasts, and its protein product is localized in the pericellular bone matrix of osteocytes. ${ }^{9}$ These findings suggest the homeostatic role of DMP1 toward mature bone formation.

Specificity of the anti-DMP1 (peptide C) polyclonal antibody used in the present study was demonstrated by the immunoblotting and immunohistochemical analyses. First, the immunoblotting showed a single band at the expected size. Second, the present immunohistochemistry showed the pericellular DMP1 immunoreactivities of osteocytes, which is compatible with our previous observation using the antibody against the N-terminus of rat DMP $1 .{ }^{9}$ In addition, the peptide absorption tests in both analyses confirmed the specificity of the antibody.

Strong DMP1 expression was observed in PMTs of all three cases, whereas other 11 soft tissue tumors never showed DMP1 expression. All of the PMTs showed positive staining for both CD34 and factor
XIIIa, whereas two malignant hemangiopericytomas and three solitary fibrous tumors were positive for CD34 and/or factor XIIIa, showing that PMTs could not be differentiated from other soft tissue tumors using these antibodies. Although PMTs were composed of heterogeneous tissues, which contained primitive-appearing stromal cells, variably prominent vessels, cartilaginous or myxomatous areas, dystrophic calcification, and microcystic changes, DMP1 was constantly detected in PMTs, indicating that DMP1 is a valuable pathological marker for PMTs. The amount of tissues from resected PMTs is typically limited in these small tumors, and therefore the diagnostic presence of ossification and bony matrix may not always be identified. In such cases, positive immunoreactivity for DMP1 in the small surgical specimens would also help in making a decision for the subsequent treatment strategy such as the confirmation of the safety margin. Furthermore, the extracellular deposition of DMP1 in PMTs will indicate the former presence of the tumors even after the tumor cells are partially denatured or have disappeared.

DMP1 accumulation in PMT was observed in the extracellular matrix as granular deposits, as well as in the tumor cells as paranuclear deposits. Under ultrastructural observation, the tumor cells of PMT were separated by an intercellular matrix containing numerous matrix granules showing early dystrophic calcification. ${ }^{4}$ Therefore, the intercellular granular deposits of DMP1 observed in the present study might be in concordance with these calcified granules.

Besides the diagnostic utility of DMP1 for PMTs, its essential role in oncogenic osteomalacia is still unknown. Among the proteins over-expressed in PMT, FGF-23 has been discussed in association with the clinical symptoms of oncogenic osteomalacia. FGF-23 induced hypophosphatemia with increased renal phosphate clearance. ${ }^{13}$ An elevated serum level of FGF-23 in patients with oncogenic osteomalacia returned to the normal range after resection of PMT. ${ }^{17}$ In some cases, the elevated 
serum FGF-23 level remained elevated even after resection of tumor, although the characteristic symptoms disappeared (unpublished data). FGF-23 might not be a sole factor that is responsible for oncogenic osteomalacia. Recently, DMP1 was detected in normal human serum by immunoblotting, ${ }^{18}$ although its serum level in patients with oncogenic osteomalacia has not been reported. Estimation of DMP1 level in the sera of the patients with oncogenic osteomalacia may provide an insight into the role of DMP1 in oncogenic osteomalacia.

In conclusion, DMP1 expression determined by immunohistochemistry could be used for the differential diagnosis of PMTs from other soft tissue tumors.

\section{Acknowledgements}

This work was supported by Grant-in-Aid 13671898 and 15591930 from the Ministry of Education, Science, and Culture of Japan, and the 21st Century COE Program.

\section{References}

1 Lyles KW. Oncogenic osteomalacia. In: Bilezikian JP, Raisz LG, Rodan GA (eds). Principles of Bone Biology, 2nd Edn. Academic Press: San Diego, 2002, pp 12091215.

2 Drezner MK. Tumor-induced osteomalacia. Rev Endocr Metab Disord 2001;2:175-186.

3 Fukumoto S, Yamashita T. Fibroblast growth factor-23 is the phosphaturic factor in tumor-induced osteomalacia and may be phosphatonin. Curr Opin Nephrol Hypertens 2002;11:385-389.

4 Weidner N, Santa Cruz D. Phosphaturic mesenchymal tumors, a polymorphous group causing osteomalaciarickets. Cancer 1987;59:1442-1454.

5 George A, Sabsay B, Simonian PAL, et al. Characterization of a novel dentin matrix acidic phosphoprotein. Implications for induction of biomineralization. J Biol Chem 1993;268:12624-12630.

6 George A, Gui J, Jenkins NA, et al. In situ localization and chromosomal mapping of the AG1 (Dmp1) gene. J Histochem Cytochem 1994;42:1527-1531.
7 D'Souza RN, Cavender A, Sunavala G, et al. Gene expression patterns of murine dentin matrix protein 1 (Dmp1) and dentin sialophosphoprotein (DSPP) suggest distinct developmental functions in vivo. J Bone Miner Res 1997;12:2040-2049.

8 MacDougall M, Gu TT, Luan X, et al. Identification of a novel isoform of mouse dentin matrix protein 1: spatial expression in mineralized tissues. J Bone Miner Res 1998;13:422-431.

9 Toyosawa S, Shintani S, Fujiwara T, et al. Dentin matrix protein 1 is predominantly expressed in chicken and rat osteocytes but not in osteoblasts. J Bone Miner Res 2001;16:2017-2026.

10 Fen JQ, Zhang J, Dallas SL, et al. Dentin matrix protein 1 , a target molecule for Cbfa1 in bone, is a unique bone marker gene. J Bone Miner Res 2002;17:1822-1831.

11 Boskey AL, Tan M, Spevak L, et al. Recombinant dentin matrix protein promotes initial in vitro mineralization. J Dent Res 2000;79S:152.

12 Narayanan K, Srinivas R, Ramachandran A, et al. Differentiation of embryonic mesenchymal cells to odontoblast-like cells by overexpression of dentin matrix protein 1. Proc Natl Acad Sci USA 2001;98:4516-4521.

13 Shimada T, Mizutani S, Muto T, et al. Cloning and characterization of FGF23 as a causative factor of tumor-induced osteomalacia. Proc Natl Acad Sci USA 2001;98:6500-6505.

14 De Beur SM, Finnegan RB, Vassiliadis J, et al. Tumors associated with oncogenic osteomalacia express genes important in bone and mineral metabolism. J Bone Miner Res 2002;17:1102-1110.

15 Tsujimura T, Sakaguchi K, Aozasa K. Phosphaturic mesenchymal tumor, mixed connective tissue variant (oncogenic osteomalacia). Pathol Int 1996;46: 238-241.

16 Kawai Y, Morimoto S, Sakaguchi K, et al. Oncogenic osteomalacia secondary to nasal tumor with decreased urinary excretion of cAMP. J Bone Miner Metab 2001;19:61-64

17 Yamazaki Y, Okazaki R, Shibata M, et al. Increased circulatory level of biologically active full-length FGF-23 in patients with hypophosphatemic rickets/osteomalacia. J Clin Endocrinol Metab 2002;87: 4957-4960.

18 Jain A, Karadag A, Fohr B, et al. Three SIBLINGs (small integrin-binding ligand, N-linked glycoproteins) enhance factor H's cofactor activity enabling MCP-like cellular evasion of complement-mediated attack. J Biol Chem 2002;277:13700-13708. 